\section{Asian/White Differences in the Relationship of Maternal Age to Low Birth Weight: Analysis of the PRAMS Survey, 2004-2011}

Asian/Pacific Island Nursing Journal Volume 1(4): 138-148

(C)Author(s) 2016

http://digitalscholarship.unlv.edu/apin/

\author{
Sangmi Kim ${ }^{\text {a }}$
}

\begin{abstract}
This study aimed to examine (a) maternal age patterns of low birth weight (LBW; birth weight $<2,500 \mathrm{~g}$ ) for non-Hispanic (N-H) Asian and N-H White women, and (b) Asian-White gaps in LBW risk by maternal age and their mechanisms. Logistic regression analyses were performed on the Pregnancy Risk Assessment Monitoring System data of N-H Asian and N-H White women who delivered their first singleton birth without birth defects in 13 states between 2004 and 2011. Age- and race/ethnicity-specific LBW risk was estimated, unadjusted and adjusted for maternal risk factors (e.g., marital status, maternal education, pregnancy intention, stress, maternal morbidities, smoking, and prenatal care) and their interactions with maternal age or race/ethnicity. The interaction between maternal age and race/ethnicity was statistically significant $(p<.0001)$ with covariates and interactions held constant. N-H Asian women showed a reverse W-shaped maternal-age pattern of LBW with the highest risk in their late 30s $(O R=1.56,95 \% \mathrm{CI}[1.26,1.94])$ whereas $\mathrm{N}-\mathrm{H}$ White women experienced a maternal age-related increase in LBW. N-H Asian women were more likely to deliver LBW infant than their N-H White counterparts between their late $20 \mathrm{~s}$ and late $30 \mathrm{~s}$, with the greatest racial/ethnic gap in their late $20 \mathrm{~s}$ ( $O R=4.19,95 \% \mathrm{CI}$ [3.33, 5.29]). Preventive strategies should be developed targeting N-H Asian women aged 25 to 39 years to reduce the Asian-White disparities in LBW. Considering the known maternal risk factors failed to explain such disparities, future research is warranted to explore other risk factors unique to this at-risk population.
\end{abstract}

Keywords: Asian, maternal age, low birth weight, disparities, weathering, PRAMS

Racial/ethnic disparities in low birth weight (LBW; birth weight $<2,500 \mathrm{~g}$ ) have persisted in the United States for decades. Black-White disparities have been well documented, due to their large gap in LBW risk. In 2012, the prevalence of LBW was highest among non-Hispanic (N-H) Black women (13.18\%), almost two-fold of N-H White women (6.97\%). The LBW prevalence among N-H Black women was followed by that among Asian/Pacific Islander women $(8.21 \%$; Martin, Hamilton, Osterman, Curtin, \& Mathews, 2013). Asian women were 30\% more likely to give birth to LBW infants than their White counterparts even after controlling for maternal age, education, nativity status, marital status, health insurance, tobacco use, gestational age, diabetes, parity, and infant's sex (Borrell, Rodriguez-Alvarez, Savitz, $\&$ Baquero, 2016).
Despite Asian women's excessive LBW risk compared to their White counterparts, less is understood about Asian-White gaps in LBW risk and mechanisms, possibly due to the lack of data. Asians are the fastest-growing population in the United States. By 2050, 33.4 million residents will identify as Asian only, representing a $213 \%$ population increase compared with a $49 \%$ increase in the total U.S. population (Islam et al., 2010). Between 1990 and 2008, the share of births for Asian women increased from 3\% to $6 \%$ (Livingston \& Cohn, 2010).

aniversity of Pennsylvania, USA

Corresponding Author:

Sangmi Kim, MPH, RN

University of Pennsylvania School of Nursing

Email: sangmik@upenn.edu 
Researchers have reported LBW risks among racial/ethnic groups as an aggregate of maternal age, masking important within-group differences in birth outcomes across maternal age (Reichman \& Pagnini, 1997). Geronimus (1992) documented that Black and White women in the reproductive period (18-49 years) have different maternal age patterns of adverse birth outcomes. Black women tend to experience a monotonic increase in adverse birth outcomes with maternal age such that Black teenagers aged 18-19 years had more favorable birth outcomes than their older counterparts. In contrast, White women showed higher adverse birth outcomes at the extremes of maternal age, with the nadir in their late 20 s to early 30 s. The linear increase in adverse birth outcomes among Black women is referenced as weathering, manifesting as an erosion of reproductive potential among Black women due to life-long stress, accumulated at advancing maternal age. As a function of the different maternal-age patterns of adverse birth outcomes between Black and White women, racial gaps widen among older women (Geronimus, 1996).

This hypothesis can be applied to other racial/ethnic minorities than Blacks who have been exposed to stressors, such as long-term socioeconomic disadvantage and discrimination (Powers, 2013). Nevertheless, no study by far has examined maternal-age patterns of adverse birth outcomes among Asian women from the weathering perspective. Therefore, this study aimed to (a) compare maternal-age distributions of LBW risk between $\mathrm{N}-\mathrm{H}$ Asian and $\mathrm{N}-\mathrm{H}$ White women, (b) examine Asian-White gaps in LBW risk by maternal age (race $\times$ maternal age interaction), and (c) investigate if several maternal risk factors and their interactions explain the race $\times$ maternal age interaction. A hypothesis specific to each study aim is as follows: Hypothesis 1: N-H Asian and N-H White women show a different maternal age pattern of LBW; Hypothesis 2: Asian-White gaps in LBW risk are different across maternal age; and Hypothesis 3: Race/ethnicity and maternal age-specific risk factors for LBW account for a maternal age pattern of LBW among N$\mathrm{H}$ Asian and $\mathrm{N}-\mathrm{H}$ White women.

\section{Method}

The Centers for Disease Control and Prevention (CDC; 2012) provided the Pregnancy Risk Assessment Monitoring System (PRAMS) data, with birth certificates appended. PRAMS, an ongoing, population-based surveillance project, monitors maternal attitudes and experiences before to shortly after pregnancy. The PRAMS projects are collaborative efforts with states. Every month each participating state selects a sample of newly delivered mothers from live birth certificates by stratified random sampling without replacement to receive a mailed questionnaire. Participating states sample between 1,300 and 3,400 women each year (CDC, 2012). The PRAMS questionnaire consists of two parts, core and standard/statedeveloped questions. The core questionnaire collects information on (a) attitudes and feelings about the most recent pregnancy, (b) content and source of prenatal care, (c) maternal alcohol and tobacco consumption, (d) physical abuse before and during pregnancy, (e) pregnancy-related morbidity, (f) infant health care, (g) contraceptive use, (h) mother's knowledge of pregnancy-related health issues (e.g., adverse effects of tobacco and alcohol), (i) benefits of folic acid, and (j) risks of HIV (CDC, 2015a). The standard/state-developed questionnaire is composed of a pretested list of standard questions developed by the CDC or developed by states on their own. As a result, each state's PRAMS questionnaire is unique (CDC, 2015b). States mail questionnaires 2 to 6 months after delivery and follow-up with a telephone interview for nonrespondents. The final PRAMS data set are weighted for sample design, nonresponse, and noncoverage to allow construction of population estimates representative of all women who gave birth in each state participating in the PRAMS during the specified years (CDC, 2012). To minimize nonresponse bias, the CDC PRAMS' working group set a response rate threshold of $65-70 \%$ (CDC, 2015a).

The data used for this study were births occurring between 2004 and 2011 from Colorado, Delaware, Florida, Georgia, Nebraska, New York, Ohio, Oregon, Pennsylvania, South Carolina, Tennessee, Washington, and Wyoming. Data from 11 states that are included in the PRAMS questionnaire were excluded from the analysis because they did not collect some key variables (e.g., diabetes before pregnancy, gestational diabetes, and smoking during pregnancy). A total of 59,423 women (7,216 Asian and 52,207 White) from the 13 states participated in the PRAMS survey. Among them, 7,238 women were excluded whose newborn was not first-order $(1,854)$, singleton $(4,607)$ birth, or had birth defects (777). Also, 19,979 women were excluded due to missing information on the birth order, plurality, and birth defects. Missing data $(11,300)$ were imputed on the dependent and independent variables by using regression method for continuous variables and logistic regression method for categorical variables because the data were assumed to have a monotone missing data pattern (Yuan, 2010). The purpose of multiple imputations in this study was to prevent loss of LBW cases among Asian women for a more accurate estimation of their LBW 
risk, considering the small sample size of this population. The final sample consisted of 5,221 N-H Asian and 38,285 N-H White women who delivered the first, singleton birth without birth defects. The study received an exemption from the review by the Institutional Review Board of the University of Pennsylvania before conducting the analysis.

\section{Dependent and Independent Variables}

The outcome variable was LBW and was defined as birth weight less than 2,500g. In the PRAMS data, birth weight is grouped into $250 \mathrm{~g}$ intervals, and the numeric value is a midpoint of the interval. Thus, birth weight was dichotomized into LBW and normal birth weight using the $2,500 \mathrm{~g}$ cut-off. Predictor variables were maternal race/ethnicity and maternal age. Race/ethnicity was determined based on women's self-report of their race and Hispanic ethnicity. Only $\mathrm{N}-\mathrm{H}$ Asian and N-H White (hereafter, Asian and White) were included in this study. In the PRAMS data, maternal age was coded in seven groups $(\leq 17$, 18-19, 20-24, 25-29, 30-34, 35-39, and 40 and older $[40+])$. Due to small cell sizes, two separate teenage groups were combined into one $(<20$ years $)$.

\section{Covariates}

The analysis incorporated maternal sociodemographic, psychological, medical, and behavioral characteristics, considered confounders or covariates in previous studies. Marital status was categorized as "married" and "others." Maternal education was categorized into five groups by years (e.g., 0-8, 9-11, 12, $13-15,16$ years or more). Pregnancy intention was dichotomized into wanted and unwanted pregnancy. Unwanted pregnancy meant women answered, "I didn't want to be pregnant then or at any time in the future." Otherwise, the pregnancy was deemed wanted. Stress was measured by stressful life events (SLEs) during the 12 months before delivery, asking women if they experienced any of the 13 events. SLEs encompass stress in multiple domains: emotional (sick/hospitalized family member, demise of someone very close), financial (job loss, difficulty paying bills), partner-related (separation or divorce, unwanted pregnancy by husband/partner), and traumatic (homelessness, imprisonment of partner/self; Lu \& Chen, 2004). The median number of SLEs was one, used as a cut-off to dichotomize the variable into lower and higher stress.

Maternal morbidity included high blood pressure (including pregnancy-induced hypertension, preeclampsia, or toxemia) during pregnancy (yes or no), diabetes before pregnancy (yes or no), gestational diabetes (yes or no), and pre-pregnancy body mass index (BMI; underweight, < 18.5; healthy weight, 18.5-
24.9; overweight, 25.0-29.9; and obesity, $\geq 30.0$; CDC, 2014). Pregnancy complications included; women with problems during pregnancy which included vaginal bleeding, urinary tract infection, severe nausea, vomiting, dehydration, cerclage for incompetent cervix, problems with the placenta, preterm or early labor, premature rupture of membrane, blood transfusion, or car accident. Initially, high blood pressure was a pregnancy complication question; instead, this was treated as a single independent covariate due to its great importance as a risk factor of $\mathrm{LBW}$ (Ødegård, Vatten, Nilsen, Salvesen, \& Austgulen, 2000). Nine pregnancy complications without high blood pressure were summed for a score ranging from 0 to 9 , categorized into $0,1,2$, and 3 or more complications, due to the small proportion of women with more than three complications at the same time during pregnancy. Health behaviors were measured by smoking during the last three months of pregnancy (yes or no), and prenatal care (PNC) received in the first trimester (yes, no, or no PNC).

\section{Statistical Analysis}

Descriptive statistics of study participants were implemented for selected variables by race/ethnicity and maternal age, using frequencies and proportions for categorical variables. Chi-square tests determined the statistical significance of the differences in participants' characteristics by race/ethnicity and maternal age. Moreover, logistic regression (using the SAS procedure PROC GENMOD with binomial distribution and logit link) was adopted to test significance of a race/ethnicity $\times$ maternal age interaction before and after adjusting for multiple covariates (marital status, maternal education, pregnancy intention, stress, maternal morbidities, pregnancy complications, health behaviors, states, and survey years) and their interactions with maternal age or race/ethnicity.

In Model 1 (main-effect model), a race/ethnicity $\times$ maternal age interaction was examined after controlling for variations among the 13 PRAMS states and survey years (2004-2011). In Model 2, the interaction was examined, holding constant all maternal risk factors, states, and survey years. In Model 3, statistically significant three-way interactions (risk factors $\times$ maternal age $\times$ race/ethnicity) were added to Model 2 to see if different maternal age distributions of the risk factors by race/ethnicity explained a maternal age trajectory of LBW unique to each racial/ethnic group. Finally, in Model 4 (interaction model), twoway interactions of all risk factors with maternal age were included to adjust for confounding of the race/ethnicity $\times$ maternal age interaction by interactions of other risk factors with maternal age. For goodness-of-fit, Akaike information criterion (AIC) index was compared among the four models; a smaller AIC 
value indicates better fit. The significance of the interaction term was determined at $p<.05$ to assess if race/ethnicity moderated the effect of maternal age on LBW risk. The PRAMS weight statement was included throughout the modeling process to account for sample selection and responses and to reflect the population of mothers delivering live births in the 13 PRAMS states during the 2004-2011 survey periods. All analyses were conducted using SAS Version 9.4 statistical software (SAS Institute, Inc., Cary, NC).

Table 1. Subject Characteristics

\begin{tabular}{|c|c|c|c|c|c|}
\hline \multirow[b]{2}{*}{ Characteristic } & \multicolumn{2}{|c|}{$\begin{array}{c}\text { Asian } \\
n=5,221\end{array}$} & \multicolumn{2}{|c|}{$\begin{array}{c}\text { White } \\
n=38,285\end{array}$} & \multirow[b]{2}{*}{$p$-value } \\
\hline & $\%$ & $S E$ & $\%$ & $S E$ & \\
\hline \multicolumn{6}{|l|}{ Maternal age (years) } \\
\hline$\leq 19$ & 2.0 & 0.4 & 7.7 & 0.2 & $<.0001$ \\
\hline $20-24$ & 9.8 & 0.8 & 21.7 & 0.3 & \\
\hline $25-29$ & 29.4 & 1.2 & 29.2 & 0.3 & \\
\hline $30-34$ & 33.9 & 1.2 & 26.1 & 0.3 & \\
\hline $35-39$ & 21.9 & 1.1 & 12.6 & 0.2 & \\
\hline $40+$ & 3.0 & 0.4 & 2.8 & 0.1 & \\
\hline \multicolumn{6}{|l|}{ Marital status } \\
\hline Married & 88.6 & 0.8 & 70.9 & 0.3 & $<.0001$ \\
\hline Others & 11.4 & 0.8 & 29.1 & 0.3 & \\
\hline \multicolumn{6}{|l|}{ Maternal education (years) } \\
\hline $0-8$ & 1.8 & 0.4 & 1.6 & 0.1 & $<.0001$ \\
\hline $9-11$ & 5.0 & 0.6 & 9.9 & 0.2 & \\
\hline 12 & 15.5 & 1.0 & 24.0 & 0.3 & \\
\hline $13-15$ & 20.8 & 1.1 & 29.4 & 0.3 & \\
\hline$\geq 16$ & 56.8 & 1.3 & 35.1 & 0.3 & \\
\hline \multicolumn{6}{|l|}{ Pregnancy intention } \\
\hline Intended & 92.6 & 0.7 & 92.0 & 0.2 & \\
\hline Not intended & 7.4 & 0.7 & 8.0 & 0.2 & \\
\hline \multicolumn{6}{|l|}{ Stress } \\
\hline Lower stress & 74.6 & 1.1 & 55.6 & 0.4 & $<.0001$ \\
\hline Higher stress & 25.4 & 1.1 & 44.4 & 0.4 & \\
\hline \multicolumn{6}{|c|}{ High blood pressure before pregnancy } \\
\hline Yes & 5.2 & 0.5 & 12.9 & 0.2 & $<.0001$ \\
\hline No & 94.8 & 0.5 & 87.1 & 0.2 & \\
\hline \multicolumn{6}{|l|}{ Diabetes before pregnancy } \\
\hline Yes & 0.9 & 0.2 & 0.8 & 0.1 & .0092 \\
\hline No & 99.1 & 0.2 & 99.2 & 0.1 & \\
\hline \multicolumn{6}{|l|}{ Gestational diabetes } \\
\hline Yes & 14.9 & 0.9 & 9.8 & 0.2 & \\
\hline No & 85.1 & 0.9 & 90.2 & 0.2 & \\
\hline \multicolumn{6}{|l|}{ BMI before pregnancy } \\
\hline Underweight & 10.0 & 0.8 & 4.7 & 0.2 & $<.0001$ \\
\hline Normal & 66.4 & 1.3 & 53.4 & 0.4 & \\
\hline Overweight & 16.6 & 1.0 & 23.0 & 0.3 & \\
\hline Obese & 7.0 & 0.7 & 19.0 & 0.3 & \\
\hline \multicolumn{6}{|c|}{ Number of pregnancy complications } \\
\hline 0 & 59.7 & 1.3 & 51.5 & 0.4 & $<.0001$ \\
\hline 1 & 28.8 & 1.2 & 28.8 & 0.3 & \\
\hline 2 & 8.2 & 0.7 & 13.8 & 0.3 & \\
\hline$\geq 3$ & 3.3 & 0.5 & 6.0 & 0.2 & \\
\hline \multicolumn{6}{|l|}{ Smoking during pregnancy } \\
\hline Yes & 9.5 & 0.7 & 20.8 & 0.3 & $<.0001$ \\
\hline No & 90.5 & 0.7 & 79.2 & 0.3 & \\
\hline \multicolumn{6}{|l|}{ Prenatal care at 1 st trimester } \\
\hline Yes & 83.2 & 1.0 & 85.8 & 0.3 & $<.0001$ \\
\hline No & 14.5 & 1.0 & 13.8 & 0.3 & \\
\hline No prenatal care & 2.3 & 0.4 & 0.4 & 0.0 & \\
\hline
\end{tabular}

Note. $\mathrm{BMI}=$ body mass index. 


\section{Results}

\section{Subject Characteristics}

Table 1 shows the distribution of characteristics for Asian $(n=5,221)$ and White $(n=38,285)$ women. More women that were Asian tended to delay childbearing to older ages than did White women. Sociodemographic and psychological risk profiles for Asian mothers were superior to those for White mothers. For example, $25.4 \%$ of Asian mothers experienced high stress compared to $44.4 \%$ of White mothers. Relative to Asian mothers, White mothers were more likely to be hypertensive during pregnancy, be overweight or obese before pregnancy, and experience at least two pregnancy complications. Asian mothers, on the other hand, were more likely than their White counterparts to experience gestational diabetes. For health behaviors, White mothers were two times more likely than their Asian counterparts to smoke during pregnancy; fewer Asian mothers received PNC in the first trimester than did White mothers.

Table 2. Distribution of Births and Risk Factors for LBW, by Maternal Age and Race/Ethnicity: First Singleton Births to Asian and White Mothers, 13 PRAMS states, 2004-2011 by Percentage ${ }^{a}$

\begin{tabular}{|c|c|c|c|c|c|c|c|}
\hline & \multirow[b]{2}{*}{$\begin{array}{l}\text { Race/ } \\
\text { Ethnicity }\end{array}$} & \multicolumn{6}{|c|}{ Maternal age (years) } \\
\hline & & $\leq 19$ & $20-24$ & $25-29$ & $30-34$ & $35-39$ & $40+$ \\
\hline \multirow[t]{2}{*}{ LBW } & White & 6.6 & 5.7 & 4.6 & 4.1 & 4.7 & 6.5 \\
\hline & Asian & 11.6 & 7.8 & 5.1 & 7.1 & 5.9 & 12.1 \\
\hline \multirow[t]{2}{*}{ Unmarried } & White & 82.9 & 54.4 & 21.7 & 11.0 & 11.3 & 13.8 \\
\hline & Asian & 67.6 & 32.8 & 11.2 & 6.1 & 6.3 & 2.6 \\
\hline \multirow[t]{2}{*}{ No high school diploma } & White & 50.8 & 18.2 & 7.7 & 3.7 & 3.1 & 2.7 \\
\hline & Asian & 44.2 & 9.9 & 7.2 & 4.5 & 5.7 & 3.9 \\
\hline \multirow[t]{2}{*}{ Unwanted pregnancy } & White & 7.8 & 8.2 & 6.6 & 7.2 & 10.3 & 19.4 \\
\hline & Asian & 14.9 & 8.8 & 5.5 & 5.6 & 9.6 & 20.4 \\
\hline \multirow[t]{2}{*}{ Higher stress } & White & 66.2 & 62.9 & 42.6 & 33.2 & 29.8 & 31.4 \\
\hline & Asian & 37.0 & 42.8 & 28.7 & 21.1 & 17.1 & 37.3 \\
\hline \multirow[t]{2}{*}{ Hypertensive during pregnancy } & White & 14.7 & 13.8 & 13.0 & 11.3 & 12.8 & 14.7 \\
\hline & Asian & 5.2 & 4.6 & 5.2 & 5.2 & 5.4 & 5.4 \\
\hline \multirow[t]{2}{*}{ Diabetes before pregnancy } & White & 0.7 & 0.8 & 0.8 & 0.6 & 1.2 & 1.4 \\
\hline & Asian & 0.0 & 0.4 & 0.3 & 1.3 & 0.9 & 3.4 \\
\hline \multirow[t]{2}{*}{ Gestational diabetes } & White & 7.9 & 9.1 & 9.3 & 10.2 & 11.2 & 15.0 \\
\hline & Asian & 7.4 & 10.4 & 13.5 & 15.9 & 17.9 & 15.8 \\
\hline \multirow[t]{2}{*}{ Overweight to obese } & White & 31.7 & 42.3 & 43.8 & 41.8 & 42.7 & 46.9 \\
\hline & Asian & 25.0 & 30.1 & 25.3 & 21.7 & 21.2 & 26.1 \\
\hline \multirow[t]{2}{*}{ Pregnancy complications } & White & 63.3 & 59.3 & 47.0 & 42.4 & 38.7 & 40.8 \\
\hline & Asian & 51.7 & 48.7 & 45.6 & 36.3 & 35.2 & 37.4 \\
\hline \multirow[t]{2}{*}{ Smoking during pregnancy } & White & 27.2 & 28.1 & 19.9 & 16.5 & 16.4 & 15.0 \\
\hline & Asian & 10.8 & 10.7 & 7.9 & 9.7 & 10.9 & 7.9 \\
\hline \multirow[t]{2}{*}{ No prenatal care at 1 st trimester } & White & 31.4 & 21.3 & 11.3 & 8.5 & 10.1 & 13.6 \\
\hline & Asian & 63.6 & 28.7 & 15.8 & 13.2 & 13.7 & 18.9 \\
\hline
\end{tabular}

Note. $\mathrm{LBW}=$ low birth weight; PRAMS = Pregnancy Risk Assessment Monitoring System; ${ }^{\mathrm{a}} p$-values from chi-square tests were significant for the listed maternal characteristics by maternal age within each racial/ethnic group.

\section{Maternal Age Distributions of $L B W$ and the Risk Factors by Race/Ethnicity}

Table 2 presents the distribution of LBW births and several risk factors for LBW by race/ethnicity and maternal age. Asian women were more likely to experience LBW than White women across maternal age. Also, maternal-age patterning differed between racial/ethnic groups: W-shape for
Asian and U-shape for White women (see Figure 1). Of the risk factors, only the maternal age trajectory of diabetes before pregnancy, gestational diabetes, and smoking during pregnancy was significantly different by race/ethnicity. Specifically, a maternal age-related increase in the prevalence of diabetes before pregnancy and gestational diabetes was steeper for Asian women than was White women. The prevalence of smoking during pregnancy decreased with maternal 
age for both racial/ethnic groups, and such a decline was greater for White women.

\section{Logistic Regression Models}

As mentioned above, four models were built to examine a race/ethnicity $\times$ maternal age interaction: Model $1=$ race/ethnicity + maternal age + race/ethnicity $\times$ maternal age + states + survey years; Model $2=$ Model $1+$ all risk factors; Model $3=$ Model $2+$ selected risk factors $\times$ race/ethnicity $\times$ maternal age; and Model $4=$ Model $3+$ all risk factors $\times$ maternal age . In Model 3, the risk factors significantly interacting with race/ethnicity and maternal age only included diabetes before pregnancy, gestational diabetes, and smoking during pregnancy. In Model 4, the risk factors in two-way interactions included all risk factors, except for those already adjusted in Model 3.

Besides, AIC considerably decreased as covariates and interactions were controlled for step-wise, showing improved model fit from the simplest (Model 1) to the most complex model (Model 4). AIC for Model 1 to Model 4 was 1254019, 1141419, 1138644, and 1131483 , respectively. The race/ethnicity $\times$ maternal age interaction in Model 4 remained statistically significant after controlling for the covariates, two-, and three-way interactions ( $p<.0001$; data not shown).

\section{Estimated Maternal Age Pattern of LBW Risk by Race/Ethnicity}

Odds ratio (OR) of LBW birth by maternal age (25-29 years as a referent group) was estimated for Asian and White women, unadjusted and adjusted for the covariates and the interactions in the main-effect model (Model 1) and the interaction model (Model 4), respectively.

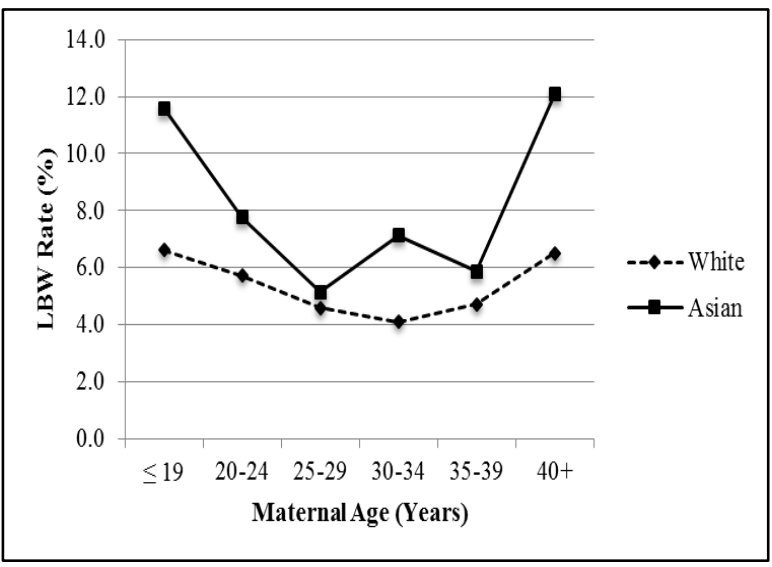

Figure 1. LBW birth rates by maternal age and race/ethnicity: 13 PRAMS states, 2004-2011.
In the main-effect model, the predicted maternal-age patterns of LBW risk were similar to those in the raw data without considering states and survey years, yielding W- and U-shaped pattern for Asian and White women, respectively. In the interaction model, however, different patterns emerged for both racial/ethnic groups. LBW risk for White women increased with maternal age, greatest between the late 20 s and early $30 \mathrm{~s}$. In contrast, LBW risk for Asian women increased with maternal age until late 20s and then plateaued. Asian women in their teens and early 20s experienced far lower LBW risk than the referent group $(p<.001)$, and the result was the same when both maternal age groups were clumped into one to ensure a larger cell size (data not shown).

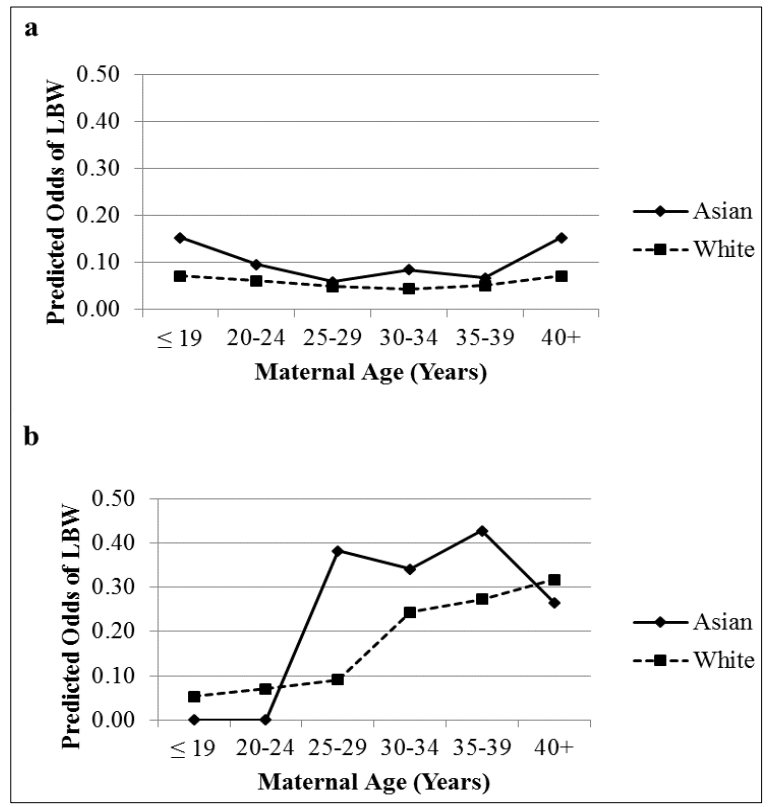

Figure 2. Predicted odds of LBW by maternal age and race/ethnicity in (a) main effect and (b) interaction models: 13 PRAMS states, 2004-2011.

\section{Estimated Asian-White Gaps in LBW Risk by Maternal Age}

Figure 2 shows the maternal age-specific odds of LBW birth for each racial/ethnic group. In the main-effect model, the Asian-White gap in LBW risk was wider when women were in their teens, early 30 s, and $40+$ years, compared to other maternal age groups. In these age periods, Asian women were approximately twice as more likely to give birth to LBW as their White counterparts. The Asian-White OR of LBW birth in teens, early 30s, and 40+ years, respectively, was 2.15 (95\% CI [1.93, 2.40]), 1.93 (95\% CI 
[1.86, 2.00]), and 2.13 (95\% CI [1.94, 2.33]; see Figure 2, Panel a).

In the interaction model, Asian women were more likely to experience LBW birth than their White counterparts between their late 20s and late 30s where $85.2 \%$ of Asian and $67.9 \%$ of White women birthed their first child. The Asian-White OR in their late 20s, early 30s, and late 30s was 4.19 (95\% CI [3.33, 5.29]), 1.40 (95\% CI $[1.21,1.62])$, and 1.56 (95\% CI [1.26, 1.94]), respectively. In contrast, Asian women were less likely to experience LBW birth than their White counterparts in their teens, early 20 s, and $40+$ years (see Figure 2, Panel b). Comparing the main effect and the interaction models, the Asian-White disparities in LBW risk decreased across maternal age, except for in their late $20 \mathrm{~s}$ and late $30 \mathrm{~s}$, after controlling for covariates and interactions.

\section{Discussion}

This would be the first study to compare maternal-age patterns of LBW risk between Asian and White women. With the same maternal-age-specific risk profiles, the two groups tended to experience a maternal age-related increase in LBW risk. Although White women presented weathering manifested as the linearly upward trend of LBW risk at advancing maternal age, Asian women showed a reverse $\mathrm{W}$-shaped pattern, with a decrease in LBW risk in their 40+.

Findings of the maternal age-related increase in LBW risk among White women as a whole adds to the existing evidence of weathering in this racial group. Divergent maternal-age patterns of poor birth outcomes by race/ethnicity were reported in the literature, dominantly among Black and White women. There was some consensus on the maternal age-related increase in adverse birth outcomes among Black women, particularly those in a more disadvantageous psychosocial environment (Collins, Simon, Jackson, \& Drolet, 2006; Geronimus, Hicken, Keene, \& Bound, 2006; Love, David, Rankin, \& Collins, 2010). Evidence of weathering among White women, however, is inconsistent across studies. Some reported that White women experienced weathering when they had Medicaid, were unmarried, living in poor neighborhoods, smoking cigarettes, or receiving inadequate PNC (de Jongh, Locke, Paul, \& Hoffman, 2012; Holzman et al., 2009; Rauh, Andrews, \& Garfinkel, 2001; Rich-Edwards, Buka, Brennan, \& Earls, 2003). Others documented no weathering among White women, regardless of their socioeconomic and smoking status (Buescher \& Mittal, 2006; Hibbs, Rankin, David, \& Collins, 2016; Sheeder, Lezottte, \& Stevens-Simon, 2006). Previous studies that argued weathering only among underprivileged White women were conducted by analyzing the limited data in terms of a sampling frame, drawing the sample population from some geographical areas. The areas included New York City (Rauh et al., 2001), Chicago (Rich-Edwards et al., 2003), or specific cities/counties in five states (Holzman et al., 2009), or 19 U.S. hospitals (de Jongh et al., 2012). This study, however, is more generalizable in that analyzing PRAMS data from 13 states, which sampled the representative populations through a stratified sampling technique at the state-level.

This study partially supported weathering among Asian women, although LBW risk abated among those aged $40+$ years. Researchers have conducted no direct investigation of weathering among Asian women in the United States mostly due to their small sample size in the collected data (Love et al., 2010; Reagan \& Salsberry, 2005). Penfield, Cheng, and Caughey (2013), however, hinted that weathering could extend to Asians because Asian adolescents had lower odds of several obstetric complications-preterm birth, primary cesarean delivery, and gestational diabetes - in comparison with N-H White adolescents. This result underpins the premise of weathering where the age of best reproductive health is younger for racial/ethnic minority women with more stress burden throughout their lives. Likewise, Asian teenagers in this study were less likely to experience these risk factors of LBW, relative to their White counterparts, including diabetes before pregnancy, gestational diabetes, and pregnancy complications.

In light of weathering, maternal age can be redefined as the duration of exposure to stressful life conditions (Rich-Edwards et al., 2003). Among immigrant populations, maternal age can also represent a length of time in the United States. Immigrant advantage - more favorable health outcomes among the foreign-born - erodes with time in the United States as women age, which is attributed to chronic stress from the process of acculturation and discrimination based on their race or language (Gee, Ro, Shariff-Marco, \& Chae, 2009; Powers, 2013). Indeed, Asian Americans had the stronger association between racial discrimination and BMI (a marker of metabolic dysfunctions in the face of prolonged stress) with increasing years in the United States (Gee, Ro, Gavin, \& Takeuchi, 2008). Similarly, the association between language discrimination and chronic health conditions was stronger for Asian American immigrants who lived in the United States for ten years or longer, relative to those who lived in the United States for less than ten years (Yoo, Gee, \& Takeuchi, 2009). Although not incorporating direct chronic stress measures or sources of chronic stress (e.g., racial or language discrimination), this study observed a greater risk of diabetes before and during pregnancy among older Asian women, signaling weather-away of Asian women's health at advancing maternal age. Presumably, the decrease in 
LBW risk in their $40+$ years could be ascribed to reserved capacity among midlife Asian women to buffer the deleterious chronic stress-effect. According to Yip, Gee, and Takeuchi (2008), middle-aged Asian Americans are less likely to experience psychological distress from racial discrimination because they have more stability in their lives and develop protective coping mechanisms.

This study reported significant interactions among maternal age, race/ethnicity, and three risk factors: diabetes before pregnancy, gestational diabetes, and smoking during pregnancy. According to the weathering hypothesis, accumulated stress may accelerate biological aging, which can lead to the earlier manifestation of chronic degenerative disease (e.g., cardiovascular or metabolic disease). Such chronic conditions would compromise women's chances of delivering a healthy infant even before they conceive the pregnancy (Geronimus, Andersen, \& Bound, 1991; Hogue \& Bremner, 2005). Also, as a response to chronic stress, women may adopt or reinforce behavioral coping mechanisms, such as smoking, drinking, or late PNC (Gavin, Nurius, \& Logan-Greene, 2012; Myers, 2009), which can elevate their adverse birth risks. Among these biomedical and behavioral pathways, hypertension and smoking have repeatedly suggested to explain weathering and the underlying chronic stress mechanism among racial/ethnic minority women (Geronimus, 1996; Holzman et al., 2009; Khoshnood, Wall, \& Lee, 2005; Powers, 2013; RichEdwards et al., 2003). Hibbs et al. (2016) even argued that smoking might serve as a proxy measure of chronic stress.

Consistent with the literature, smoking rates during pregnancy decreased with maternal age among White women in this study (Geronimus, Neidert, \& Bound, 1993; Rich-Edwards et al., 2003). Asian women, however, did not report increasing hypertension or smoking rates with maternal age as observed among Black women (Geronimus et al., 1991; Miranda et al., 2010). Instead, Asian and White women experienced increased diabetes before pregnancy and gestational diabetes at advancing maternal age, which was steeper among Asian women. Because wear and tear of the body in response to chronic stress may lead to cardiovascular or metabolic system disturbance (Juster, McEwen, \& Lupien, 2010), diabetic conditions, not hypertension, may significantly contribute to weathering in Asian and White women. Also, as seen among Asian and White women in this study, an opposite direction of maternal age trajectory of LBW from that of smoking during pregnancy was observed among U.S.-born Hispanic women. Wildsmith (2002) reported that U.S.-born Hispanic women who experienced weathering in neonatal mortality showed an age pattern of smoking, highest around age 18 years and declining steadily after that. The Asian-White disparities in LBW, not entirely explained by the comprehensive maternal risk factors and their interactions with race/ethnicity or maternal age, may imply that other driving force of weathering than the known risk factors (e.g., smoking) could exist for Asian and White women.

\section{Limitations}

The limitations to this study included the small sample size of Asian women may have produced a less precise estimate of LBW risk, particularly for teens. Due to the same reason, heterogeneity of the Asian population could not be considered but could have been achieved through separate analysis according to subethnicities (e.g., Chinese, Japanese, Filipino, and other Asians). Wong et al. (2008) did report a variation of mean birth weight among nine Asian subethnic groups at a national level in 2003: Chinese $(3,275 \mathrm{~g})$, Japanese $(3,161 \mathrm{~g})$, Filipino $(3,186 \mathrm{~g})$, Asian Indian $(3,132 \mathrm{~g})$, Korean $(3,302 \mathrm{~g})$, Vietnamese $(3,186 \mathrm{~g})$, Samoan $(3,537 \mathrm{~g})$, Guamanian $(3,210 \mathrm{~g})$, and Hawaiian $(3,274 \mathrm{~g})$. Second, not all PRAMS states collected such variables as racial discrimination, neighborhood safety, chronic stress, nativity, acculturation, and duration of U.S. residence, although they may play an essential role in weathering among racial/ethnic minority women (Buescher \& Mittal, 2006; Collins, Rankin, \& Hedstrom, 2012; Geronimus, 1996; Love et al., 2010; Miranda et al., 2010; Powers, 2013; Wildsmith, 2002). Only a few states selectively collected racial discrimination or nativity, but the data from 13 states between 2004 and 2011 did not contain these variables. Finally, failing to differentiate foreign-born Asian women from their U.S.-born counterparts may have confounded the real picture of the maternal-age relationship with LBW birth in this population.

\section{Conclusion}

The purpose of this study was to examine a maternal age trajectory of Asian-White differences in LBW and the underlying mechanisms. The study findings provided evidence that weathering of reproductive potential, culminating into LBW, can occur among not only Black but also White and Asian women with maternal age. However, the unexplained Asian-White disparities in LBW by race/ethnicity and maternal age-specific risk profiles point to the existence of more proximate risk factor of weathering, such as chronic stress.

Based on these findings, this paper concludes with the following suggestions for future research and practice to alleviate the Asian-White disparities in LBW. First, studies should be replicated in other Asian 
and White populations to confirm maternal-age patterns of LBW. Also, greater effort is necessary to reveal weathering mechanisms. Although chronic stress has been suggested to drive weathering, few studies empirically examined chronic-stress pathways to explicate the relationships among maternal age, race/ethnicity, and birth outcomes. That SLEs did not explain the differences in LBW risk between Asian and White women may suggest the need to collect and develop chronic stress measures that consider racial/ethnic uniqueness in stress experience. Finally, considering the differential extent in the Asian-White gap by maternal age, targeted strategies should be developed to reach the specific races/ethnicities and age groups of women. For Asian women in their teens, early 20s, and $40+$ years, modification of known maternal risk factors could reduce LBW risk. In particular, early PNC should be more accessible to Asian teenagers. To reduce excessive LBW risk among Asian women aged between their late 20 s and late 30 s, health care personnel should carefully monitor gestational diabetes through screening in these age groups. Also, risk factors unique to Asian women between their late 20s and late 30 s should be explored.

\section{Acknowledgments}

Data from the Pregnancy Risk Assessment Monitoring System (PRAMS) included in this study are collected at the state level by the following state collaborators and their staff, as represented by the PRAMS Working Group: Colorado (Alyson Shupe, $\mathrm{PhD}$ ), Delaware (George Yocher, MS), Florida (Alisa Simon), Georgia (Qun Zheng, MS), Nebraska (Brenda Coufal), New York State (Anne Radigan), Ohio (Connie Geidenberger, PhD), Oregon (Claudia W. Bingham, MPH), Pennsylvania (Tony Norwood), South Carolina (Mike Smith, MSPH), Tennessee (Ramona Lainhart, PhD), Washington (Linda Lohdefinck), and Wyoming (Amy Spieker, MPH); CDC PRAMS Team, Applied Sciences Branch, Division of Reproductive Health.

\section{Declaration of Conflicting Interest}

The author has no disclosures to make.

\section{Funding}

The study was funded by the Xi Chapter of Sigma Theta Tau.

\section{References}

Borrell, L. N., Rodriguez-Alvarez, E., Savitz, D. A., \& Baquero, M. C. (2016). Parental race/ethnicity and adverse birth outcomes in New York City: 20002010. American Journal of Public Health, 106, 1491-1497. doi:10.2105/AJPH.2016.303242

Buescher, P. A., \& Mittal, M. (2006). Racial disparities in birth outcomes increase with maternal age: Recent data from North Carolina. North Carolina Medical Journal, 67, 16-20.

Centers for Disease Control and Prevention. (2012). PRAMS methodology. Retrieved from http://www.cdc.gov/prams/methodology.htm

Centers for Disease Control and Prevention. (2014). Recommended BMI-for-age cutoffs. Retrieved from http://www.cdc.gov/nccdphp/dnpao/growthcharts /training/bmiage/page4.html

Centers for Disease Control and Prevention. (2015a). Indicator definitions-mental health. Retrieved from http://www.cdc.gov/cdi/definitions/mentalhealth.html

Centers for Disease Control and Prevention. (2015b). PRAMS questionnaires. Retrieved from http://www.cdc.gov/prams/questionnaire.htm

Collins, J. W., Rankin, K. M., \& Hedstrom, A. B. (2012). Exploring weathering: The relation of age to low birth weight among first generation and established United States-born Mexican-American women. Maternal and Child Health Journal, 16, 967-972. doi:10.1007/s10995-011-0827-4

Collins, J. W., Jr., Simon, D. M., Jackson, T. A., \& Drolet, A. (2006). Advancing maternal age and infant birth weight among urban African Americans: The effect of neighborhood poverty. Ethnicity \& Disease, 16, 180-186. Retrieved from http://ishib.orgwww.ishib.org/ED/journal/16-1/ethn-16-01180.pdf

de Jongh, B. E., Locke, R., Paul, D. A., \& Hoffman, M. (2012). The differential effects of maternal age, race/ethnicity and insurance on neonatal intensive care unit admission rates. BMC Pregnancy and Childbirth, 12, Art. 97. doi:10.1186/1471-239312-97

Gavin, A. R., Nurius, P., \& Logan-Greene, P. (2012). Mediators of adverse birth outcomes among socially disadvantaged women. Journal of Women's Health, 21, 634-642. doi:10.1089/jwh.2011.2766

Gee, G. C., Ro, A., Gavin, A., \& Takeuchi, D. T. (2008). Disentangling the effects of racial and weight discrimination on body mass index and obesity among Asian Americans. American Journal of Public Health, 98, 493-500. doi: 10.2105/AJPH.2007.114025

Gee, G. C., Ro, A., Shariff-Marco, S., \& Chae, D. (2009). Racial discrimination and health among Asian Americans: Evidence, assessment, and directions for future research. Epidemiologic Reviews, 31, 130-151. doi: 10.1093/epirev/mxp009 
Geronimus, A. T. (1992). The weathering hypothesis and the health of African-American women and infants: Evidence and speculations. Ethnicity \& Disease, 2, 207-221.

Geronimus, A. T. (1996). Black/white differences in the relationship of maternal age to birthweight: A population-based test of the weathering hypothesis. Social Science \& Medicine, 42, 589-597. doi:10.1016/0277-9536(95)00159-X

Geronimus, A. T., Andersen, H. F., \& Bound, J. (1991). Differences in hypertension prevalence among U.S. Black and White women of childbearing age. $P u b-$ lic Health Reports, 106, 393-399.

Geronimus, A. T., Hicken, M., Keene, D., \& Bound, J. (2006). "Weathering" and age patterns of allostatic load scores among Blacks and Whites in the United States. American Journal of Public Health, 96, 826-833. doi:10.2105/AJPH.2004.060749

Geronimus, A. T., Neidert, L. J., \& Bound, J. (1993). Age patterns of smoking in US Black and White women of childbearing age. American Journal of Public Health, 83, 1258-1264. doi:10.2105/AJPH.83.9.1258

Hibbs, S., Rankin, K. M., David, R. J., \& Collins, J. W. (2016). The relation of neighborhood income to the age-related patterns of preterm birth among White and African-American women: The effect of cigarette smoking. Maternal and Child Health Journal, 20, 1432-1440. doi:10.1007/s10995016-1941-0

Hogue, C. J., \& Bremner, J. D. (2005). Stress model for research into preterm delivery among Black women. American Journal of Obstetrics and Gynecology, 192, S47-55. doi:10.1016/j.ajog.2005.01.073

Holzman, C., Eyster, J., Kleyn, M., Messer, L. C., Kaufman, J. S., Laraia, B. A., ... Elo, I. T. (2009). Maternal weathering and risk of preterm delivery. American Journal of Public Health, 99, 1864-1871. doi:10.2105/AJPH.2008.151589

Islam, N. S., Khan, S., Kwon, S., Jang, D., Ro, M., \& TrinhShevrin, C. (2010). Methodological issues in the collection, analysis, and reporting of granular data in Asian American populations: Historical challenges and potential solutions. Journal of Health Care for the Poor and Underserved, 21, 13541381. doi:10.1353/hpu.2010.0939

Juster, R. P., McEwen, B. S., \& Lupien, S. J. (2010). Allostatic load biomarkers of chronic stress and impact on health and cognition. Neuroscience and Biobehavioral Reviews, 35, 2-16. doi:10.1016/j.neubiorev.2009.10.002

Khoshnood, B., Wall, S., \& Lee, K. S. (2005). Risk of low birth weight associated with advanced maternal age among four ethnic groups in the United States. Maternal and Child Health Journal, 9, 3-9. doi:10.1007/s10995-005-2446-4

Livingston, G., \& Cohn, D. (2010). The new demography of American motherhood. Retrieved from http://www.pewsocialtrends.org/2010/05/06/thenew-demography-of-american-motherhood/
Love, C., David, R. J., Rankin, K. M., \& Collins, J. W. (2010). Exploring weathering: Effects of lifelong economic environment and maternal age on low birth weight, small for gestational age, and preterm birth in African-American and White women. American Journal of Epidemiology, 172, 127-134. doi:10.1093/aje/kwq109

Lu, M. C., \& Chen, B. (2004). Racial and ethnic disparities in preterm birth: The role of stressful life events. American Journal of Obstetrics and Gynecology, 191, 691-699. doi:10.1016/j.ajog.2004.04.018

Martin, J., Hamilton, B., Osterman, M., Curtin, S., \& Mathews, T. (2013). Births: Final data for 2012. National Vital Statistics Reports, 62(9). Retrieved from http://www.cdc.gov/nchs/data/nvsr/nvsr62/nvsr62 -09.pdf

Miranda, M. L., Swamy, G. K., Edwards, S., Maxson, P., Gelfand, A., \& James, S. (2010). Disparities in maternal hypertension and pregnancy outcomes: Evidence from North Carolina, 1994-2003. Public Health Reports, 125, 579-587.

Myers, H. F. (2009). Ethnicity- and socio-economic-statusrelated stresses in context: An integrative review and conceptual model. Journal of Behavioral Medicine, 32, 9-19.

Ødegård, R. A., Vatten, L. J., Nilsen, S. T., Salvesen, K. Å., and Austgulen, R. (2000). Preeclampsia and fetal growth. Obstetrics \& Gynecology, 96, 950-955. doi:10.1016/S0029-7844(00)01040-1

Penfield, C. A., Cheng, Y. W., \& Caughey, A. B. (2013). Obstetric outcomes in adolescent pregnancies: A racial/ethnic comparison. The Journal of Maternal-Fetal \& Neonatal Medicine, 26, 1430-1434. doi:10.3109/14767058.2013.784738

Powers, D. A. (2013). Paradox revisited: A further investigation of racial/ethnic differences in infant mortality by maternal age. Demography, 50, 495-520. doi:10.1007/s13524-012-0152-6

Rauh, V. A., Andrews, H. F., \& Garfinkel, R. S. (2001). The contribution of maternal age to racial disparities in birthweight: A multilevel perspective. American Journal of Public Health, 91, 1815-1824. doi:10.2105/AJPH.91.11.1815

Reagan, P. B., \& Salsberry, P. J. (2005). Race and ethnic differences in determinants of preterm birth in the USA: Broadening the social context. Social Science \& Medicine, 60, 2217-2228.

Reichman, N. E., \& Pagnini, D. L. (1997). Maternal age and birth outcomes: Data from New Jersey. Family Planning Perspectives, 29, 268-272, 295. doi:10.2307/2953415

Rich-Edwards, J. W., Buka, S. L., Brennan, R. T., \& Earls, F. (2003). Diverging associations of maternal age with low birthweight for Black and White mothers. International Journal of Epidemiology, 32, 83-90. doi:10.1093/ije/dyg008

Sheeder, J., Lezottte, D., \& Stevens-Simon, C. (2006). Maternal age and the size of White, Black, Hispanic, and mixed infants. Journal of Pediatric and Adolescent Gynecology, 19, 385-389. doi:10.1016/j.jpag.2006.09.012 
Wildsmith, E. M. (2002). Testing the weathering hypothesis among Mexican-origin women. Ethnicity \& Disease, 12, 470-479. Retrieved from http://www.ishib.org/journal/ethn-12-04-470.pdf

Wong, L. F., Caughey, A. B., Nakagawa, S., Kaimal, A. J., Tran, S. H., \& Cheng, Y. W. (2008). Perinatal outcomes among different Asian-American subgroups. American Journal of Obstetrics \& Gynecology, 199, 382 e381-386. doi: 101016/j.ajog.2008.06.073

Yip, T., Gee, G. C., \& Takeuchi, D. T. (2008). Racial discrimination and psychological distress: The impact of ethnic identity and age among immigrant and United States-born Asian adults.
Developmental Psychology, 44, 787-800. doi: 10.1037/0012-1649.44.3.787

Yoo, H. C., Gee, G. C., \& Takeuchi, D. (2009). Discrimination and health among Asian American immigrants: Disentangling racial from language discrimination. Social Science \& Medicine, 68, 726732 .

doi:http://dx.doi.org/10.1016/j.socscimed.2008.1 1.013

Yuan, Y. C. (2010). Multiple imputation for missing data: Concepts and new developments (Version 9.0). Retrieved from http://citeseerx.ist.psu.edu/viewdoc/down-

load?doi=10.1.1.306.5728\&rep=rep1\&type=pdf 